Meta

Journal des traducteurs

Translators' Journal

\title{
Noyaux conceptuels et traduction médicale
}

\section{Sylvie Vandaele}

Volume 46, numéro 1, mars 2001

Traduction médicale et documentation / Medical translation and documentation

URI : https://id.erudit.org/iderudit/004533ar

DOI : https://doi.org/10.7202/004533ar

Aller au sommaire du numéro

Éditeur(s)

Les Presses de l'Université de Montréal

ISSN

0026-0452 (imprimé)

1492-1421 (numérique)

Découvrir la revue

Citer cet article

Vandaele, S. (2001). Noyaux conceptuels et traduction médicale. Meta, 46(1), 16-21. https://doi.org/10.7202/004533ar

\section{Résumé de l'article}

Les cours de traduction spécialisée ne peuvent prendre en charge l'enseignement de l'ensemble des notions composant un domaine. Nous proposons d'extraire des domaines de spécialité des noyaux conceptuels constitués, par exemple, des principes sur lesquels se fonde une discipline ou de tout autre élément fondamental susceptible de servir de base d'évolution d'une discipline. Ces noyaux conceptuels, qui seront enseignés au cours de la formation du traducteur, serviront à ce dernier de fondements cognitifs dans son domaine de spécialité. Ils lui serviront d'ancrages auxquels relier les notions nouvelles rencontrées dans les textes à traduire, lui permettant de structurer logiquement ses recherches documentaires et ainsi de transformer progressivement ses doutes en éléments de compétence s'enrichissant avec le temps.
Ce document est protégé par la loi sur le droit d'auteur. L'utilisation des services d’Érudit (y compris la reproduction) est assujettie à sa politique d'utilisation que vous pouvez consulter en ligne.

https://apropos.erudit.org/fr/usagers/politique-dutilisation/ 


\title{
Noyaux conceptuels et traduction médicale
}

\author{
SYLVIE VANDAELE \\ Université de Montréal, Montréal, Canada
}

\begin{abstract}
RÉSUMÉ
Les cours de traduction spécialisée ne peuvent prendre en charge l'enseignement de l'ensemble des notions composant un domaine. Nous proposons d'extraire des domaines de spécialité des noyaux conceptuels constitués, par exemple, des principes sur lesquels se fonde une discipline ou de tout autre élément fondamental susceptible de servir de base d'évolution d'une discipline. Ces noyaux conceptuels, qui seront enseignés au cours de la formation du traducteur, serviront à ce dernier de fondements cognitifs dans son domaine de spécialité. Ils lui serviront d'ancrages auxquels relier les notions nouvelles rencontrées dans les textes à traduire, lui permettant de structurer logiquement ses recherches documentaires et ainsi de transformer progressivement ses doutes en éléments de compétence s'enrichissant avec le temps.
\end{abstract}

\section{ABSTRACT}

A translation course cannot cover all the concepts of a particular field. We propose to extract and provide the student with conceptual cores, for example the fundamental principles of a discipline or any other basic information. The acquisition of these conceptual cores during a translation course should establish a cognitive basis on which future knowledge will be built. They will be used as references to which new concepts found in texts to be translated should be linked, allowing translators to design logical search strategies and to replace, over time, uncertainty with an ever-increasing wealth of knowledge.

\section{MOTS-CLÉS/KEYWORDS}

Noyaux conceptuels, enseignement de la traduction spécialisée, recherches documentaires, fondements cognitifs, notions nouvelles.

Une question particulièrement épineuse revient régulièrement quand il s'agit de définir la matière enseignée dans le cadre d'un cours de traduction médicale: jusqu'où faut-il aller dans l'enseignement des notions? Celles-ci sont complexes et la médecine, au cours des cinquante dernières années, a considérablement élargi son champ, puisqu'elle dépasse les classiques domaines de l'anatomie et de la physiologie pour se nourrir à des disciplines connexes qui lui étaient autrefois étrangères, telles que la génétique, la biologie moléculaire, la biochimie, la biologie cellulaire, la physique, la chimie, mais aussi les statistiques ou l'éthique... Or, s'il est bien entendu qu'un cours de traduction n'est pas un cours de médecine ou de biologie, on sait également que la compréhension d'un texte, phase essentielle à une traduction réussie, tous les autres paramètres mis à part, nécessite de posséder un certain bagage cognitif. Ainsi, C. Balliu reprend «les observations judicieuses de Gile (1986: 26), selon lesquelles un texte scientifique se fonde sur des "contenus cognitifs spécialisés" que ne connaissent que les spécialistes» (Balliu 1994: 17). C'est précisément le point essentiel: un spécialiste, pour mettre à jour ses connaissances (ce qu'il ne manque pas de faire continuellement), rattache les nouveaux concepts à un bagage cognitif déjà organisé. Il en est de même, d'ailleurs, pour un traducteur d'expérience spécialisé dans un domaine particulier.

Meta, XLVI, 1, 2001 
Qu'en est-il lorsqu'un apprenti-traducteur ou un traducteur a devant lui un texte traitant d'un domaine de connaissance qui lui est étranger? L'analyse préliminaire de protocoles de verbalisation ${ }^{1}$ (voir Dancette 1995 pour la méthode utilisée) que nous avons réalisée sur un extrait de texte spécialisé traitant de biologie moléculaire montre la difficulté d'extraire le sens - même quand une documentation adéquate est fournie au sujet - s'il ne connaît que très peu le domaine. D'une part, la consultation de la documentation de référence, si celle-ci traite d'un sujet complexe, est ardue, notamment si le temps alloué au travail de traduction est limité (ce qui est le cas dans la réalité la plupart du temps). Ainsi, le sujet manifeste fréquemment son incompréhension et même son découragement devant la complexité des ouvrages de référence. D'autre part, les définitions consultées restent des éléments quelque peu disparates, en partie parce qu'elles font appel elles-mêmes à d'autres notions spécialisées. Il apparaît donc clairement que faute d'ancrage à un bagage cognitif minimal préexistant, le sujet éprouve la plus grande difficulté à saisir le fil logique qui pourrait lui permettre de répondre efficacement aux questions qu'il se pose sur le texte. La prise de décision quant à la traduction elle-même se situe essentiellement sur le plan lexical et sur la pertinence de l'usage de tel ou tel terme, mais un certain nombre de questions ou d'hypothèses formulées quant au sens restent sans réponse. Par conséquent, le traducteur conserve un certain degré d'incertitude quant à la validité de sa traduction. Enfin, le temps passé à l'analyse du texte n'est pas entièrement rentabilisé: les connaissances acquises au cours du processus restent fragmentaires et ne sont pas organisées en un ensemble cohérent qui pourrait servir à un ancrage ultérieur. Il est fort possible qu'avec le temps et de la persévérance, les doutes et les questions finissent par se résoudre, mais les contraintes extérieures ne le permettront peut-être pas. En résumé, notre analyse, bien que préliminaire, met en évidence la difficulté que pose la compréhension d'un texte complexe en l'absence d'une stratégie faisant appel à un ancrage dans un bagage cognitif déjà constitué.

Ces résultats n'ont rien d'étonnant, car l'usage des termes dépend de la structure cognitive dans laquelle ils s'insèrent, ainsi que Sager (1990: 75) l'a déjà souligné: «It is important to realise that terms can only be used if the user already possesses the configuration of knowledge which determines the role of the term in a structured system.» Quelles sont alors les solutions auxquelles il est possible de recourir? Les bases de connaissances terminologiques qui, grâce à l'utilisation de réseaux sémanticoterminologiques, contiennent "pour chaque terme une forte dose d'informations conceptuelles hautement structurées» (Otman 1997: 251) peuvent en être une. Toutefois, si ces outils formels sont de toute évidence, tant pour l'apprenti-traducteur que pour le traducteur en exercice, d'une utilité majeure, deux écueils subsistent: d'une part, ils sont encore rares et, d'autre part, dans un cadre pédagogique, le problème est d'une autre nature qui va au-delà de la question terminologique. Comme le montre l'analyse préliminaire des protocoles de verbalisation évoquée plus haut, la question est celle de l'ancrage du raisonnement devant le texte à traduire. En son absence, il est difficile de relier les concepts contenus dans le texte de manière organisée, par exemple, en établissant une carte des concepts ${ }^{2}$. Par conséquent, notre hypothèse de travail est que, pour utiliser avec profit des outils tels que les ouvrages de référence et les bases de connaissance terminologiques (outils externes au texte à traduire), ainsi que les cartes de concepts (outils permettant de travailler sur le texte lui-même), il est nécessaire d'ancrer le raisonnement à une structure cognitive 
préexistante. Certes, l'idée de favoriser chez l'apprenant la construction «d'une architecture de connaissances » à laquelle il fera appel lorsqu'il se verra placé devant des problèmes à résoudre n'est certes pas nouvelle; elle constitue l'un des apports fondamentaux de la psychologie cognitive à la pédagogie (Tardif 1997). Mais quelle forme lui donner dans le cadre de l'enseignement de la traduction spécialisée?

Nous établissons la structure cognitive qui servira d'ancrage au raisonnement et à la prise de décision traductionnels comme étant un noyau conceptuel. En l'état actuel de notre réflexion, nous en donnons la définition suivante: il s'agit d'un ensemble de concepts autour desquels s'organise un domaine notionnel. Dans cette définition, «concept» est entendu au sens «d'abstraction pouvant jouer le rôle d'une unité au sein d'une théorie» (Armstrong 1987:228), et il est entendu que, contrairement à la terminologie, un concept ne correspond pas nécessairement à un terme unique.

Quelle forme donner à un noyau conceptuel? À titre d'exemple, nous présenterons la structure du noyau conceptuel de la pharmacologie, laquelle est «l'étude des médicaments et de leur emploi » (Manuila et Nicoulin 1995: 310). Or, la pharmacologie moderne se fonde essentiellement sur l'idée que les substances médicamenteuses agissent sur différentes structures au sein de l'organisme. Le noyau conceptuel de la pharmacologie occidentale moderne pourrait donc se formuler ainsi:

1. Les substances pharmacologiquement actives ont la propriété d'interagir de manière plus ou moins spécifique avec des molécules cibles de l'organisme.

2. Ces molécules-cibles participent au fonctionnement des cellules qui constituent le tissu des organes composant l'organisme.

3. Les substances pharmacologiquement actives modifient donc les processus physiologiques de l'organisme, ce qui est censé permettre à celui-ci de lutter contre la maladie.

Comme on peut le voir, définir le noyau conceptuel relatif à la pharmacologie revient, dans ce cas, à dégager les éléments qui fondent la discipline. Ce faisant, il est possible de souligner les termes importants et les liens qu'ils entretiennent entre eux, d'une part, et avec d'autres noyaux conceptuels, d'autre part. Nous concrétiserons ces relations par un schéma (Figure 1). Ainsi, on aborde les constituants de l'organisme par l'intermédiaire de notions qu'il est possible de classer hiérarchiquement: «organisme», «organes», «tissu», «cellules», «molécules». Chacun de ces termes permet de faire le lien avec les noyaux conceptuels de domaines connexes: anatomie, physiologie, pathologie, biologie cellulaire, biologie moléculaire, etc. Le cœur du noyau conceptuel proposé pour la pharmacologie est ici «l'interaction plus ou moins spécifique», qui constitue un élément clé de l'activité du "médicament». Celui-ci permet de faire le lien avec les sciences pharmaceutiques et les domaines intervenant en aval, jusqu'à la commercialisation du produit. Chacun des domaines présentés dans le schéma fait lui-même, par ailleurs, l'objet de l'élaboration d'un noyau conceptuel spécifique. On complétera l'énoncé du noyau conceptuel par une série de termes sélectionnés dans les langues de départ et d'arrivée (termes essentiels ou présentant des difficultés particulières), des exemples de phraséologie et de la documentation pertinente. Les notions spécifiques à la pharmacologie qui seront vues ultérieurement dans le cadre de la traduction des textes pourront se rattacher au noyau conceptuel proposé, ce qui permettra à l'étudiant-traducteur d'enrichir progressivement le schéma cognitif qu'il se construit sur cette base en reliant les notions nouvelles qu'il rencontrera à un bagage cognitif déjà logiquement structuré. 
Figure 1: Liens existant entre les composantes du noyau conceptuel de la pharmacologie et les autres sous-domaines biomédicaux

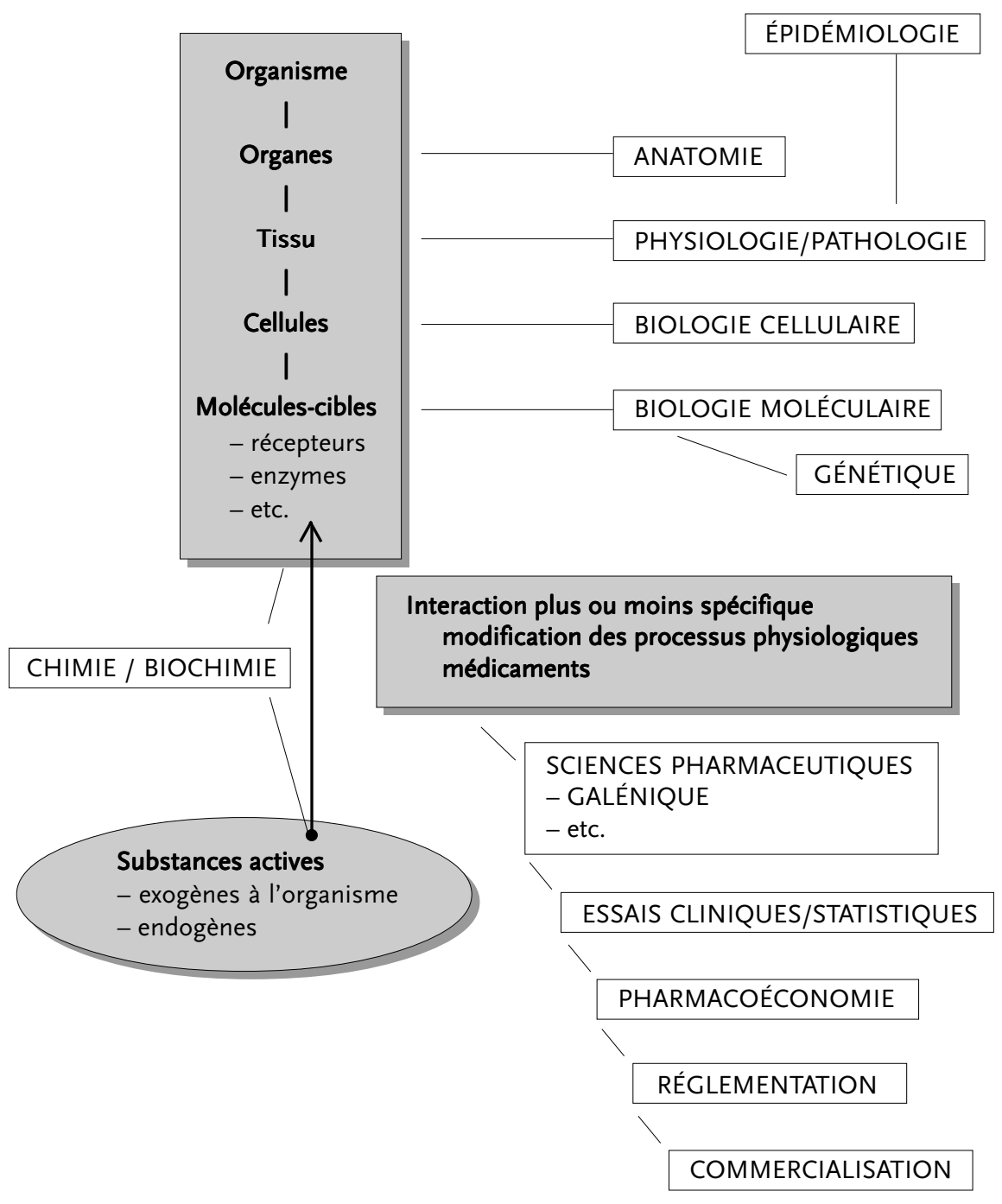

Quels pourraient être les noyaux conceptuels utilisables dans le domaine médical et comment les organiser dans un cours de traduction? Nous proposons de structurer l'ensemble des noyaux conceptuels selon un axe qui rendrait compte d'un parcours logique réalisé depuis l'organisme sain jusqu'à la mise en marché du médicament. Une telle stratégie conduit à établir sept modules théoriques, chacun faisant l'objet de la construction d'un noyau conceptuel:

1. L'organisme sain: biologie cellulaire, physiologie, anatomie

2. L'organisme malade: pathologie, épidémiologie

3. Qu'est-ce que le médicament: pharmacologie

4. Stratégies de conception du médicament: chimie, biologie moléculaire 
5. Mise en forme du médicament et voies d'administration: sciences pharmaceutiques

6. Essais cliniques et statistiques biomédicales

7. Pharmacoéconomie et gestion de la santé

L'élaboration d'un noyau conceptuel ne fait nullement appel à une automatisation quelconque de recherche réalisée à partir d'un corpus. C'est d'ailleurs à la fois sa force et sa faiblesse: il fait plutôt intervenir la connaissance d'un domaine par un expert, lequel peut être en mesure de définir les liens existant entre, par exemple, les principes fondateurs de deux disciplines connexes. Ainsi, il est bien évident que le noyau conceptuel proposé pour la pharmacologie n'est pas indépendant de celui de la chimie ou de la physiologie. Par conséquent, l'élaboration d'un noyau conceptuel dans un but pédagogique relève d'une connaissance de nature encyclopédique qui nécessite de la part du professeur soit une connaissance approfondie du domaine, soit la collaboration d'un expert. Cela ne constitue pas, à notre sens, un obstacle majeur, dans la mesure où le noyau conceptuel est essentiellement un point de départ et où le recours à ce type d'outil vise à susciter l'autonomie de la démarche réflexive chez l'apprenant, tout en facilitant le travail de l'enseignant. En effet, l'utilisation raisonnée des noyaux conceptuels dans le cadre d'un cours de traduction spécialisée devrait permettre d'enclencher d'emblée le processus d'acquisition dynamique des connaissances qui ne cessera plus tout au long de la carrière du traducteur. Par conséquent, le point de départ n’a pas besoin d'être défini de manière immuable. Enfin, l'étudiant devrait être en mesure de faire le lien entre le noyau conceptuel du domaine et le réseau de termes du texte, ce qui devrait faciliter la consultation du matériel de référence et des outils formels de terminologie. D’ailleurs, les noyaux conceptuels ne visent pas à remplacer les arbres de domaine organisés de manière hiérarchique, mais plutôt à les compléter en fournissant des liens. En ce sens, ils constituent un outil qui complète la panoplie de l'enseignant et du traducteur.

Des travaux ultérieurs devraient permettre de valider ces hypothèses: en effet, si, comme nous le supposons, le recours aux noyaux conceptuels permet de faciliter le travail de l'étudiant, mais aussi du traducteur, il devrait être possible de mettre en évidence, notamment à l'aide de protocoles de verbalisation, que le recours à un ancrage du raisonnement sur le plan des notions est facilité et que la structuration logique de la recherche d'informations devrait s'accroître. Par conséquent, le processus de compréhension devrait s'en trouver facilité et on devrait pouvoir en relever certains indices, en particulier la capacité de "délittéraliser» la traduction, une aptitude accrue à la paraphrase et une prise de décision qui ne se fonde plus uniquement sur une analyse lexicale et syntaxique, mais bien sur une analyse notionnelle. Enfin, l'ensemble du processus devrait mener le traducteur, mais plus particulièrement l'étudiant qui se heurte parfois de manière douloureuse à l'incertitude et au stress infligés par la nécessité de prendre rapidement des décisions, à consolider ses connaissances, à éliminer le doute qui reste stérile s'il n'est pas combattu par des stratégies cognitives efficaces, bref, à devenir progressivement, lui aussi, un expert dans son domaine de spécialité.

\section{NOTES}

1. Dans le cadre d'une étude pilote, nous avons proposé de traduire la phrase «We used the polymerase chain reaction to identify a mouse testis cDNA that represented another member of a growing class of mammalian endoproteases involved in the processing of precursor proteins.» (Nakayama et al. 
1992: 5897) à des traducteurs qui n'avaient pas de connaissances approfondies dans le domaine biomédical.

2. «Une carte de concepts est une représentation graphique hiérarchisée, externe, de l'organisation interne des informations d'un texte ou d'un domaine dans la mémoire d'un sujet.» (Nguyen et Tochon 1998: 3)

\section{RÉFÉRENCES}

Armstrong, D. M. (1987): "Concept», Le cerveau, un inconnu. Dictionnaire encyclopédique (R. L. Gregory, dir.), trad. de l'anglais par J. Doubovetzky, Paris, Robert Laffont, p. 228-230.

Balliu, C. (1994): "L'enseignement de la traduction médicale: pour une nouvelle pragmatique», Meta, 39-1, p. 15-27.

Dancette, J. (1995) : Parcours de traduction. Étude expérimentale du processus de traduction, Lille, Presses universitaires de Lille.

GiLe, D. (1986) : «La traduction médicale doit-elle être réservée aux seuls médecins? Quelques réflexions ", Meta, 31-1, p. 26-30.

Manuila, A. et L., et M. Nicoulin (1995): Dictionnaire médical, Paris, Masson.

NaKayama, N. et al. (1992): "Identification of the Fourth Member of the Mammalian Endoprotease Family Homologous to the Yeast Kex2 Protease," Journal of Biological Chemistry, 267-5, p. 5897-5900.

Nguyen, T. C. P. et F. V. Tochon (1998): «Influence comparée de la carte de concepts et du résumé sur la compréhension et la production orales durant l'interprétation consécutive», Meta, 42-2, p. 1-16.

Otman, G. (1997) : «Les bases de connaissance terminologiques: les banques de terminologie de seconde génération ", Meta, 42-2, p. 244-256.

Sager, J. C. (1990): A Practical Course in Terminology Processing, Amsterdam, John Benjamins.

TARdif, J. (1997): Pour un enseignement stratégique, Montréal, Éditions Logiques. 